\title{
Graded 2-Absorbing Submodules over Non-Commutative Rings
}

\author{
RASHID ABU-DAWWAS \\ Yarmouk University \\ Department of Mathematics \\ Irbid \\ JORDAN
}

\author{
HEBA SHASHAN \\ Yarmouk University \\ Department of Mathematics \\ Irbid \\ JORDAN
}

\author{
AFNAN DAGHER \\ Yarmouk University \\ Department of Mathematics \\ Irbid \\ JORDAN
}

Abstract: Let $R$ be a non-commutative $G$-graded ring with unity and $M$ be a $G$-graded left $R$-module. We define the concept of a graded 2-absorbing submodule and show that if the ring is commutative, then the concept is the same as the original definition of that of K. Al-Zoubi and R. Abu-Dawwas. We give an example to show that in general these two concepts are different. Many properties of graded 2 -absorbing submodules are introduced which aresimilartotheresultsforcommutativerings.

Key-Words: Graded 2-absorbing submodules, graded 2-absorbing ideals, graded prime submodules, graded prime ideals.

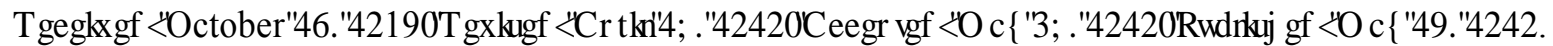

\section{Introduction}

The study of graded rings comes into being normally out of the study of affine schemes and admits them to establish and consolidate arguments by induction. However, this is not just an algebraic deception. The concept of grading in algebra, in particular graded modules is fundamental in the study of homological aspect of rings. Plenty of the contemporary growth of the commutative algebra give preference to graded rings. Graded rings play a principal role in algebraic geometry and commutative algebra. Gradings come into sight in many circumstances, both in elementary and advanced level. In recent years, rings with a group-graded structure have become progressively substantial and as a consequence, the graded analogues of various concepts are extensively studied.

In 2016, Naghani and Moghimi in [7] proposed the concept of graded 2-absorbing ideals of commutative rings with unity, which is a generalization of graded prime ideals, and investigated some properties. They defined a graded 2 -absorbing ideal $P$ of a commutative ring $R$ with unity to be a proper graded ideal of $R$ and if whenever $x, y, z \in h(R)$ with $x y z \in P$, then $x y \in P$ or $y z \in P$ or $x z \in P$. In 2019, Al-Zoubi, Abu-Dawwas and Çeken in [4] introduced several properties concerning graded 2absorbing ideals over a commutative ring with unity. Al-Zoubi and Abu-Dawwas in [3] extended graded 2absorbing ideals to graded 2-absorbing submodules over a commutative ring with unity. They defined a proper graded $R$-submodule $N$ of $M$ over a commutative ring $R$ with unity to be graded 2-absorbing if whenever $a, b \in h(R)$ and $m \in h(M)$ such that $a b m \in N$, then either $a m \in N$ or $b m \in N$ or $a b \in(N: R M)=\{r \in R: r M \subseteq N\}$. One can see that graded 2 -absorbing submodules are generalization of graded prime submodules. Furthermore, it is recognizable that graded 2-absorbing ideals are special cases of graded 2-absorbing submodules.

In recent years, the study of the graded absorbing property of graded rings, graded modules and related concepts have been some of the topics of interest in the development of the graded ring and graded module theory. In this article, we study the concept of graded 2-absorbing submodules over non-commutative rings. Also, we introduce the concept of graded strong 2absorbing submodules and show that in general if $R$ is not a commutative ring, then the concepts of graded 2 -absorbing and graded strong 2-absorbing submodules are not the same (Example 6). If $R$ is commutative, then the concept of graded 2-absorbing submodules coincides with that of the original definition introduced by Al-Zoubi and Abu-Dawwas in [3].

Graded prime submodules and graded prime ideals over non-commutative ring have been studied by Abu-Dawwas, Bataineh and Al-Muanger in [2]. A proper graded $R$-submodule $N$ of $M$ is said to be graded prime if whenever $x \in h(R)$ and $m \in h(M)$ such that $x R m \subseteq N$, then $m \in N$ or $x \in\left(N:_{R} M\right)$. A graded $R$-module $M$ is said to be graded prime if $\{0\}$ is a graded prime $R$-submodule of $M$. Also, in [2], a proper graded $R$-submodule $N$ of a graded $R$ module $M$ is said to be graded total prime if whenever $x \in h(R)$ and $m \in h(M)$ such that $x m \in N$, then either $m \in N$ or $x \in\left(N:_{R} M\right)$. Moreover, $M$ is said 
to be graded total prime if $\{0\}$ is a graded total prime $R$-submodule of $M$. In general, a graded $R$-module $M / N$ is a graded total prime $R$-module if and only if $N$ is a graded total prime $R$-submodule of $M$.

In this article, we follow [6] to introduce the concept of graded strong 2-absorbing submodules. Among several results, we prove that if $N$ is a graded prime (graded total prime) $R$-submodule of $M$, then $N$ is a graded 2-absorbing (graded strong 2 -absorbing) $R$-submodule of $M$ (Proposition 8), on the other hand, we prove that the converse is not true in general (Example 15). However, we prove that if $N$ is a graded semi-commutative 2absorbing $R$-submodule of $M$, then $N$ is a graded strong 2-absorbing $R$-submodule of $M$ (Proposition 11). Also, we show that if $N_{1}$ and $N_{2}$ are two graded prime (graded total prime) $R$-submodules of $M$, then $N_{1} \bigcap N_{2}$ is a graded 2-absorbing (graded strong 2absorbing) $R$-submodule of $M$ (Proposition 14). We prove that if $N$ and $K$ are graded $R$-submodules of $M$ such that $N \subseteq K$ and $N$ is a graded 2absorbing (graded strong 2-absorbing) $R$-submodule of $M$, then $N$ is a graded 2-absorbing (graded strong 2 -absorbing) $R$-submodule of $K$ (Proposition 16). We prove that if $N$ and $K$ are graded $R$-submodules of $M$ such that $K \nsubseteq N$ and $N$ is a graded 2-absorbing (graded strongly 2-absorbing) $R$-submodule of $M$, then $N \cap K$ is a graded 2-absorbing (graded strongly 2 -absorbing) $R$-submodule of $K$ (Proposition 21). Finally, we prove that if $K$ is a graded $R$-submodule of $M$ and $N$ is an $R$-submodules of $M$ such that $K \subseteq N$, then $N$ is a graded 2-absorbing (graded strongly 2 -absorbing) $R$-submodule of $M$ if and only if $N / K$ is a graded 2-absorbing (graded strongly 2absorbing) $R$-submodule of $M / K$ (Proposition 23).

\subsection{Preliminaries}

All rings in this article are associative (not necessarily commutative) and all modules are left $R$-modules. Let $G$ be a group with identity $e$ and $R$ be a ring. Then $R$ is said to be $G$-graded if $R=\bigoplus_{g \in G} R_{g}$ with $R_{g} R_{h} \subseteq R_{g h}$ for all $g, h \in G$, where $R_{g}$ is an additive subgroup of $R$ for all $g \in G$. The elements of $R_{g}$ are called homogeneous of degree $g$. Consider $\operatorname{supp}(R, G)=\left\{g \in G: R_{g} \neq 0\right\}$. If $x \in R$, then $x$ can be written as $\sum_{g \in G} x_{g}$, where $x_{g}$ is the component of $x$ in $R_{g}$. Moreover, $R_{e}$ is a subring of $R$ and if $R$ contains a unity 1 , then $1 \in R_{e}$. Furthermore, $h(R)=\bigcup_{g \in G} R_{g}$.

Let $I$ be an ideal of a graded ring $R$. Then $I$ is said to be a graded ideal if $I=\bigoplus_{g \in G}\left(I \cap R_{g}\right)$, i.e., for $x \in I, x=\sum_{g \in G} x_{g}$, where $x_{g} \in I$ for all $g \in G$. The following example shows that an ideal of a graded ring need not be graded.

Example 1. Consider $R=M_{2}(K)$ (the ring of all $2 \times 2$ matrices with entries from a field $K)$ and $G=$ $\mathbf{Z}_{4}$ (the group of integers modulo 4 ). Then $R$ is $G$ graded by

$$
\begin{gathered}
R_{0}=\left(\begin{array}{cc}
K & 0 \\
0 & K
\end{array}\right), R_{2}=\left(\begin{array}{cc}
0 & K \\
K & 0
\end{array}\right) \text { and } \\
R_{1}=R_{3}=\{0\} .
\end{gathered}
$$

Consider the ideal $I=\left\langle\left(\begin{array}{ll}1 & 1 \\ 1 & 1\end{array}\right)\right\rangle$ of $R$. Note that, $\left(\begin{array}{ll}1 & 1 \\ 1 & 1\end{array}\right) \in I$ such that $\left(\begin{array}{ll}1 & 1 \\ 1 & 1\end{array}\right)=$ $\underbrace{\left(\begin{array}{ll}1 & 0 \\ 0 & 1\end{array}\right)}_{\in R_{0}}+\underbrace{\left(\begin{array}{ll}0 & 1 \\ 1 & 0\end{array}\right)}_{\in R_{2}}$. If I is a graded ideal of $R$, then $\left(\begin{array}{ll}1 & 0 \\ 0 & 1\end{array}\right) \in I$ which is a contradiction. So, $I$ is not graded ideal of $R$.

Let $R$ be a $G$-graded ring and $I$ be a graded ideal of $R$. Then $R / I$ is $G$-graded by $(R / I)_{g}=\left(R_{g}+I\right) / I$ for all $g \in G$. If $R$ and $S$ are two $G$-graded rings, then $R \times S$ is $G$-graded by $(R \times S)_{g}=R_{g} \times S_{g}$ for all $g \in G$.

Assume that $M$ is a left $R$-module. Then $M$ is said to be $G$-graded if $M=\bigoplus_{g \in G} M_{g}$ with $R_{g} M_{h} \subseteq$ $M_{g h}$ for all $g, h \in G$, where $M_{g}$ is an additive subgroup of $M$ for all $g \in G$. The elements of $M_{g}$ are called homogeneous of degree $g$. Also, we consider $\operatorname{supp}(M, G)=\left\{g \in G: M_{g} \neq 0\right\}$. It is clear that $M_{g}$ is an $R_{e}$-submodule of $M$ for all $g \in G$. Moreover, $h(M)=\bigcup_{g \in G} M_{g}$.

Let $N$ be an $R$-submodule of a graded $R$-module $M$. Then $N$ is said to be a graded $R$-submodule if $N=\bigoplus_{g \in G}\left(N \cap M_{g}\right)$, i.e., for $x \in N, x=\sum_{g \in G} x_{g}$, where $x_{g} \in N$ for all $g \in G$. Similarly, as in Example 1 , an $R$-submodule of a graded $R$-module need not be graded. 


\section{Graded 2-Absorbing Submodules and Graded strong 2-Absorbing Submodules}

In this section, we study the concept of graded 2absorbing submodules over non-commutative rings. Also, we introduce and study the concept of graded strong 2-absorbing submodules.

Definition 2. Let $R$ be a graded ring and $P$ be a proper graded ideal of $R$. Then $P$ is said to be a graded 2-absorbing ideal of $R$ if whenever $x, y, z \in$ $h(R)$ such that $x R y R z \subseteq P$, then either $x y \in P$ or $y z \in P$ or $x z \in P$.

Definition 3. Let $M$ be a graded $R$-module and $N$ be a proper graded $R$-submodule of $M$. Then $N$ is said to be a graded 2-absorbing $R$-submodule of $M$ if whenever $x, y \in h(R)$ and $m \in h(M)$ such that $x R y R m \subseteq N$, then either $x m \in N$ or $y m \in N$ or $x y \in\left(N:_{R} M\right)$. A graded $R$-module $M$ is said to be graded 2-absorbing if $\{0\}$ is a graded 2-absorbing $R$-submodule of $M$.

Remark 4. If $R$ is a commutative ring, then the concept of a graded 2-absorbing $R$-submodules coincides with that of Al-Zoubi and Abu-Dawwas in [3].

Definition 5. Let $M$ be a graded R-module and $N$ be a proper graded $R$-submodule of $M$. Then $N$ is said to be a graded strong 2-absorbing $R$-submodule of $M$ if whenever $x, y \in h(R)$ and $m \in h(M)$ such that xym $\in N$, then either $x m \in N$ or ym $\in N$ or $x y \in\left(N:_{R} M\right)$. A graded R-module $M$ is said to be graded strong 2-absorbing if $\{0\}$ is a graded strong 2 -absorbing $R$-submodule of $M$.

The next example shows that if $R$ is not commutative ring, then the concepts of graded 2-absorbing and graded strong 2-absorbing submodules are not the same.

Example 6. Let $R=M_{2}(\mathbf{Z})$ (the ring of all $2 \times 2$ matrices with integer entries) and $M=$ $\left\{\left(\begin{array}{ll}0 & 0 \\ 0 & 0\end{array}\right),\left(\begin{array}{ll}1 & 1 \\ 0 & 0\end{array}\right),\left(\begin{array}{ll}0 & 0 \\ 1 & 1\end{array}\right),\left(\begin{array}{ll}1 & 1 \\ 1 & 1\end{array}\right)\right\}$ where the entries of the matrices in $M$ are from the ring $\mathbf{Z}_{2}$. Then $M$ is an $R$-module. Suppose that $G=\mathbf{Z}_{4}$. Then $R$ is $G$-graded by $R_{0}=\left(\begin{array}{cc}\mathbf{Z} & 0 \\ 0 & \mathbf{Z}\end{array}\right)$, $R_{2}=\left(\begin{array}{cc}0 & \mathbf{Z} \\ \mathbf{Z} & 0\end{array}\right)$ and $R_{1}=R_{3}=\{0\}$. Also, $M$ is $G$-graded by $M_{0}=M$ and $M_{1}=M_{2}=M_{3}=\{0\}$. The possible graded nontrivial proper $R$-submodules of $M$ are $N=\left\{\left(\begin{array}{ll}0 & 0 \\ 0 & 0\end{array}\right),\left(\begin{array}{ll}1 & 1 \\ 0 & 0\end{array}\right)\right\}$,
$K=\left\{\left(\begin{array}{ll}0 & 0 \\ 0 & 0\end{array}\right),\left(\begin{array}{ll}0 & 0 \\ 1 & 1\end{array}\right)\right\}$ and $L=$ $\left\{\left(\begin{array}{ll}0 & 0 \\ 0 & 0\end{array}\right),\left(\begin{array}{ll}1 & 1 \\ 1 & 1\end{array}\right)\right\}$ that are not closed under multiplication by $R$; for $N:\left(\begin{array}{ll}1 & 0 \\ 1 & 0\end{array}\right) \in R$ such that $\left(\begin{array}{ll}1 & 0 \\ 1 & 0\end{array}\right)\left(\begin{array}{ll}1 & 1 \\ 0 & 0\end{array}\right) \notin N$, for $K$ : $\left(\begin{array}{ll}0 & 1 \\ 0 & 1\end{array}\right) \in R$ such that

$$
\left(\begin{array}{ll}
0 & 1 \\
0 & 1
\end{array}\right)\left(\begin{array}{ll}
0 & 0 \\
1 & 1
\end{array}\right) \notin K \text { and for } L \text { : }
$$

$\left(\begin{array}{ll}1 & 0 \\ 0 & 0\end{array}\right) \in R$ such that

$$
\left(\begin{array}{ll}
1 & 0 \\
0 & 0
\end{array}\right)\left(\begin{array}{ll}
1 & 1 \\
1 & 1
\end{array}\right) \notin L \text {. So, } M \text { has no }
$$
graded nontrivial proper $R$-submodules, i.e., $M$ is a graded simple and hence $M$ is a graded prime $R$ module and as such graded 2-absorbing. On the other hand, $x=\left(\begin{array}{ll}0 & 0 \\ 0 & 3\end{array}\right), y=\left(\begin{array}{ll}0 & 3 \\ 5 & 0\end{array}\right) \in h(R)$ and $m=\left(\begin{array}{ll}0 & 0 \\ 1 & 1\end{array}\right) \in h(M)$ such that xym $=$ $\left(\begin{array}{ll}0 & 0 \\ 0 & 0\end{array}\right), x m=\left(\begin{array}{ll}0 & 0 \\ 1 & 1\end{array}\right) \neq\left(\begin{array}{ll}0 & 0 \\ 0 & 0\end{array}\right), y m=$ $\left(\begin{array}{ll}1 & 1 \\ 0 & 0\end{array}\right) \neq\left(\begin{array}{ll}0 & 0 \\ 0 & 0\end{array}\right)$ and $x y M \neq\left\{\left(\begin{array}{ll}0 & 0 \\ 0 & 0\end{array}\right)\right\}$ since $\left(\begin{array}{ll}1 & 1 \\ 1 & 1\end{array}\right) \in M$ such that $x y\left(\begin{array}{ll}1 & 1 \\ 1 & 1\end{array}\right)=$ $\left(\begin{array}{ll}0 & 0 \\ 1 & 1\end{array}\right) \neq\left(\begin{array}{ll}0 & 0 \\ 0 & 0\end{array}\right)$. So, $M$ is not graded strong 2-absorbing $R$-module.

Lemma 7. Let $M$ be a $G$-graded $R$-module and $N$ be a graded prime $R$-submodule of $M$. If $x, y \in h(R)$ and $m \in h(M)$ such that $x R y R m \subseteq N$ and $x m \notin$ $N$, then $y \in(N: R M)$.

Proof: Firstly, we show that $y R m \subseteq N$. Let $r \in$ $R$. Then $r=\sum_{g \in G} r_{g}$ where $r_{g} \in R_{g}$ for all $g \in G$, and then for $g \in G, x R\left(y r_{g} m\right) \subseteq x R(y R m) \subseteq N$. Since $N$ is graded prime, either $y r_{g} m \in N$ or $x \in$ $(N: R M)$. Then $y r_{g} m \in N$ because $x m \notin N$. That is $y R_{g} m \subseteq N$ for all $g \in G$, which implies that $y R m \subseteq N$. Since $N$ is graded prime, either $m \in N$ or $y \in\left(N:_{R} M\right)$. Since $x m \notin N$, we have $m \notin N$, so that $y \in\left(N:_{R} M\right)$.

Proposition 8. Let $N$ be a graded prime (graded total prime) $R$-submodule of $M$. Then $N$ is a graded 2absorbing (graded strong 2-absorbing) $R$-submodule of $M$. 
Proof: Suppose that $N$ is a graded prime $R$ submodule of $M$. Let $x, y \in h(R)$ and $m \in h(M)$ such that $x R y R m \subseteq N$. Assume that $x m \notin N$. Then by Lemma 7, we have $y \in\left(N:_{R} M\right)$. Then $y m \in N$ and $x y M \subseteq x N \subseteq N$. Hence, $N$ is a graded 2absorbing $R$-submodule of $M$. Now, suppose that $N$ is a graded total prime $R$-submodule of $M$. Let $x, y \in h(R)$ and $m \in h(M)$ such that $x y m \in N$. Assume that $y m \notin N$. Since $N$ is graded total prime, we have $x \in\left(N:_{R} M\right)$. Hence, $x y M \subseteq x M \subseteq N$, and we have $N$ is a graded strong 2-absorbing $R$ submodule of $M$.

Remark 9. We will prove in Example 15 that the converse of Proposition 8 is not true in general, even if the ring $R$ is commutative.

Definition 10. Let $M$ be a graded $R$-module and $N$ be a graded $R$-submodule of $M$. Then $N$ is said to be a graded semi-commutative $R$-submodule of $M$ if whenever $x \in h(R)$ and $m \in h(M)$ such that $x m \in$ $N$, then $x R m \subseteq N$.

Proposition 11. Let $M$ be a graded $R$-module and $N$ be a proper graded semi-commutative $R$-submodule of $M$. If $N$ is a graded 2-absorbing $R$-submodule of $M$, then $N$ is a graded strong 2-absorbing $R$ submodule of $M$.

Proof: Let $x, y \in h(R)$ and $m \in h(M)$ such that $x y m \in N$. Since $N$ is graded semi-commutative, we have $x R y R m \subseteq N$. Since $N$ is graded 2-absorbing, we have $x m \in N$ or $y m \in N$ or $x y M \subseteq N$. Hence, $N$ is a graded strong 2-absorbing $R$-submodule of $M$.

Compare the next Proposition with Theorem 2.5(ii) in [3].

Proposition 12. Let $M$ be a $G$-graded $R$-module and $N$ be a graded $R$-submodule of $M$. If $N_{g}$ is a 2absorbing $R_{e}$-submodule of $M_{g}$ for some $g \in G$, then $\left(N_{g}:_{R_{e}} M_{g}\right)$ is a 2-absorbing ideal of $R_{e}$.

Proof: Let $x, y, z \in R_{e}$ such that $x R_{e} y R_{e} z \subseteq$ $\left(N_{g}:_{R_{e}} M_{g}\right)$ and suppose that $x z \notin\left(N_{g}:_{R_{e}} M_{g}\right)$ and $y z \notin\left(N_{g}:_{R_{e}} M_{g}\right)$. We prove that $x y \in\left(N_{g}: R_{e}\right.$ $\left.M_{g}\right)$. Since $x z, y z \notin\left(N_{g}::_{R_{e}} M_{g}\right)$, there exist $m, s \in M_{g}$ such that $x z m \notin N_{g}$ and $y z s \notin N_{g}$. Now, $x R_{e} y R_{e} z(m+s) \subseteq N_{g}$. Since $N_{g}$ is 2-absorbing, we have $x y \in\left(N_{g}: R_{e} M_{g}\right)$ or $x z(m+s) \in N_{g}$ or $y z(m+s) \in N_{g}$. If $x z(m+s) \in N_{g}$, then $x z s \notin N_{g}$ since $x z m \notin N_{g}$. Since $x R_{e} y R_{e} z s \subseteq N_{g}$ and $y z s \notin N_{g}$ and $x z s \notin N_{g}$, we have $x y \in\left(N_{g}: R_{e}\right.$ $\left.M_{g}\right)$. Similar to the case $y z(m+s) \in N_{g}$, we obtain $x y \in\left(N_{g}: R_{e} M_{g}\right)$. Hence, $\left(N_{g}: R_{e} M_{g}\right)$ is a 2-absorbing ideal of $R_{e}$.
Lemma 13. ([5], Lemma 2.1) Let $M$ be a graded $R$ module. If $N$ and $K$ are graded $R$-submodules of $M$, then $N \cap K$ is a graded $R$-submodule of $M$.

Proof: Clearly, $N \cap K$ is an $R$-submodule of $M$. Let $m \in N \cap K$. Then $m \in N$ and $m \in K$. Since $N$ and $K$ are graded $R$-submodules of $M$, we have $m_{g} \in N$ and $m_{g} \in K$ for all $g \in G$, which implies that $m_{g} \in N \cap K$ for all $g \in G$. Hence, $N \cap K$ is a graded $R$-submodule of $M$.

Compare the next Proposition with Theorem 2.4 in [3].

Proposition 14. If $N_{1}$ and $N_{2}$ are two graded prime (graded total prime) $R$-submodules of a graded $R$ module $M$, then $N_{1} \cap N_{2}$ is a graded 2-absorbing (graded strong 2-absorbing) $R$-submodule of $M$.

Proof: By Lemma 13, we have $N_{1} \bigcap N_{2}$ is a graded $R$-submodule of $M$. If $N_{1}=N_{2}$, then $N_{1} \bigcap N_{2}$ is a graded prime (graded total prime) $R$-submodule of $M$, so that $N_{1} \cap N_{2}$ is a graded 2-absorbing (graded strong 2-absorbing) $R$-submodule of $M$. Assume that $N_{1}$ and $N_{2}$ are distinct. Since $N_{1}$ and $N_{2}$ are proper graded $R$-submodules of $M$, it follows that $N_{1} \cap N_{2}$ is a proper graded $R$-submodule of $M$. Let $x, y \in$ $h(R)$ and $m \in h(M)$ such that $x R y R m \subseteq N_{1} \bigcap N_{2}$ $\left(x y m \in N_{1} \cap N_{2}\right)$ with $x m \notin N_{1} \bigcap N_{2}$ and $x y M \nsubseteq$ $N_{1} \cap N_{2}$. Then we have the following two situations:

1. $x m \notin N_{1}$ or $x m \notin N_{2}$.

2. $x y M \nsubseteq N_{1}$ or $x y M \nsubseteq N_{2}$.

These two situations give the following four cases:

1. $x m \notin N_{1}$ and $x y M \nsubseteq N_{1}$.

2. $x m \notin N_{1}$ and $x y M \nsubseteq N_{2}$.

3. $x m \notin N_{2}$ and $x y M \nsubseteq N_{1}$.

4. $x m \notin N_{2}$ and $x y M \nsubseteq N_{2}$.

Let $N_{1}$ and $N_{2}$ be two graded prime $R$-submodules of a graded $R$-module $M$. Firstly, we consider Case (1). Since $x R y R m \subseteq N_{1} \cap N_{2} \subseteq N_{1}$ and $x m \notin N_{1}$, it follows from Lemma 7 that $y M \subseteq N_{1}$, a contradiction since $x y M \nsubseteq N_{1}$. So, Case (1) is impossible. Similarly, Case (4) does not occur. Now, we consider Case (2). Again, we get that $y M \subseteq N_{1}$ and then $y m \in$ $N_{1}$. Let $r \in R$. Then $r=\sum_{g \in G} r_{g}$ where $r_{g} \in R_{g}$ for all $g \in G$. Since $x R y R m \subseteq N_{1} \cap N_{2} \subseteq N_{2}$, we have for $g \in G, x R y r_{g} m \subseteq N_{2}$, which implies that $x M \subseteq N_{2}$ or $y r_{g} m \in N_{2}$ since $N_{2}$ is graded prime. If $x M \subseteq N_{2}$, then $x y M \subseteq x M \subseteq N_{2}$ contradicts $x y M \nsubseteq N_{2}$. Thus $y r_{g} m \in N_{2}$ for all $g \in G$ and 
then $y r m \in N_{2}$, that is $y R m \subseteq N_{2}$. Since $N_{2}$ is graded prime, we have $y M \subseteq N_{2}$ or $m \in N_{2}$. If $y M \subseteq N_{2}$, then $x y M \subseteq N_{2}$ leading to the same contradiction. Hence, $m \in N_{2}$ and then $y m \in N_{2}$. Therefore, ym $\in N_{1} \cap N_{2}$. The proof of Case (3) is similar to that of Case (2).

Let $N_{1}$ and $N_{2}$ be graded total prime $R$ submodules of $M, x, y \in h(R)$ and $m \in h(M)$ such that xym $\in N_{1} \bigcap N_{2} \subseteq N_{1}$. Consider Case (1), since $N_{1}$ is graded total prime, we have $x M \subseteq N_{1}$ or $y m \in$ $N_{1}$. If $x M \subseteq N_{1}$, then $x y M \subseteq x M \subseteq N_{1}$ which is impossible. So, $y m \in N_{1}$ and then $y M \subseteq N_{1}$ or $m \in N_{1}$ which is impossible. Therefore, Case (1) does not happen. Similarly, Case (4) does not occur. Consider Case (2), we have xym $\in N_{1} \bigcap N_{2} \subseteq N_{2}$ and since $N_{2}$ is graded total prime, we have $x M \subseteq N_{2}$ or $y m \in N_{2}$. If $x M \subseteq N_{2}$, then $x y M \subseteq x M \subseteq N_{2}$ that is a contradiction. So, $y m \in N_{2}$. Since $x y m \subseteq$ $N_{1} \cap N_{2} \subseteq N_{1}$, we have $x M \subseteq N_{1}$ or $y m \in N_{1}$. Since $x m \notin N_{1}, x M \subseteq N_{1}$ is impossible. Hence, $y m \in N_{1} \cap N_{2}$. The proof of Case (3) is similar to that of Case (2).

The next example shows that the converse of Proposition 8 is not true in general, even if the ring $R$ is commutative.

Example 15. Let $R=\mathbf{Z}, M=\mathbf{Z}_{\mathbf{6}}[\mathbf{i}]$ and $G=\mathbf{Z}_{\mathbf{2}}$. Then $R$ is G-graded by $R_{0}=\mathbf{Z}$ and $R_{1}=\{0\}$. Also, $M$ is G-graded by $M_{0}=\mathbf{Z}_{6}$ and $M_{1}=i \mathbf{Z}_{6}$. Now, $N=\{0\}$ is a graded $R$-submodule of $M$. Note that $2 \in h(R)$ and $3 \in h(M)$ such that $2.3 \in N$, but $3 \notin N$ and $2 \notin\left(N:_{R} M\right)=6 \mathbf{Z}$. Therefore, $N$ is not a graded prime (graded total prime) $R$-submodule of $M$. On the other hand, $2,3 \in h(M)$, $N_{1}=\langle 2\rangle$ and $N_{2}=\langle 3\rangle$ are graded prime (graded total prime) $R$-submodules of $M$, and by Proposition $14, N=N_{1} \bigcap N_{2}$ is a graded 2-absorbing (strong 2 -absorbing) $R$-submodule of $M$.

Proposition 16. Let $N$ and $K$ be two graded $R$ submodules of a graded $R$-module $M$ such that $N \subseteq$ $K$. If $N$ is a graded 2-absorbing (graded strong 2absorbing) $R$-submodule of $M$, then $N$ is a graded 2absorbing (graded strong 2-absorbing) $R$-submodule of $K$.

Proof: If $K=M$, then we are done. Suppose that $K \neq M$. Let $x, y \in h(R)$ and $m \in h(K)$ such that $x R y R m \subseteq N(x y m \in N)$. Since $m \in h(K), m \in$ $K_{g}=K \bigcap M_{g}$ for some $g \in G$, so $m \in h(M)$. As $N$ is a graded 2-absorbing (graded strong 2-absorbing) $R$-submodule of $M$, we have $x m \in N$ or $y m \in N$ or $x y \in\left(N:_{R} M\right)$. Since $N \subseteq K$, we have $\left(N:_{R}\right.$ $M) \subseteq(N: R K)$, and then $x m \in N$ or $y m \in N$ or $x y \in(N: R K)$. Hence, $N$ is a graded 2-absorbing (graded strong 2-absorbing) $R$-submodule of $K$.
Compare the next Lemma with Theorem 2.5(i) in [3].

Lemma 17. Let $M$ be a $G$-graded $R$-module and $N$ be a graded $R$-submodule of $M$. Assume that $g \in G$ such that $N_{g}$ is a 2-absorbing (strong 2-absorbing) $R_{e}$-submodule of $M_{g}$. Then $x R_{e} y K \subseteq N_{g}(x y K \subseteq$ $\left.N_{g}\right)$ implies that $x y \in\left(N_{g}: R_{e} M_{g}\right)$ or $x K \subseteq N_{g}$ or $y K \subseteq N_{g}$ for every $x, y \in R_{e}$ and $R_{e}$-submodule $K$ of $M_{g}$.

Proof: Suppose that $x y \notin\left(N_{g}:_{R_{e}} M_{g}\right), x K \nsubseteq N_{g}$ and $y K \nsubseteq N_{g}$. Then there exist $m, s \in K$ such that $x m \notin N_{g}$ and $y s \notin N_{g}$. Since $x R_{e} y R_{e} m \subseteq$ $x R_{e} y K \subseteq N_{g}\left(x y m \in x y K \subseteq N_{g}\right)$ and $x y \notin$ $\left(\begin{array}{lll}N_{g} & :_{R_{e}} & M_{g}\end{array}\right)$ and $x m \notin N_{g}$, we have $y m \in N_{g}$. Also, since $x R_{e} y R_{e} s \subseteq x R_{e} y K \subseteq N_{g}$ (xys $\in$ $\left.x y K \subseteq N_{g}\right)$ and $x y \notin\left(N_{g}:_{R_{e}} M_{g}\right)$ and $y s \notin N_{g}$, we have $x s \in N_{g}$. Now, since $x R_{e} y R_{e}(m+s) \subseteq$ $x R_{e} y K \subseteq N_{g}\left(x y(m+s) \in x y K \subseteq N_{g}\right)$ and $x y \notin\left(N_{g}:_{R_{e}} M_{g}\right)$, we have $x(m+s) \in N_{g}$ or $y(m+s) \in N_{g}$. If $x(m+s) \in N_{g}$, then since $x s \in N_{g}$, we have $x m \in N_{g}$ which is a contradiction. If $y(m+s) \in N_{g}$, then since $y m \in N_{g}$, we have $y s \in N_{g}$ which is a contradiction. Thus, $x y \in\left(N_{g}:_{R_{e}} M_{g}\right)$ or $x K \subseteq N_{g}$ or $y K \subseteq N_{g}$.

Proposition 18. Let $M$ be a $G$-graded $R$-module and $N$ be a graded $R$-submodule of $M$. Assume that $g \in$ $G$ such that $N_{g}$ is a 2-absorbing $R_{e}$-submodule of $M_{g}$. Then $\left(N_{g}: R_{e} R_{e} m\right)$ is a 2-absorbing ideal of $R_{e}$ for every $m \in M_{g}-N_{g}$.

Proof: Let $x, y, z \in R_{e}$ and $m \in M_{g}-N_{g}$ such that $x R_{e} y R_{e} z \subseteq\left(\begin{array}{lll}N_{g} & : R_{e} & R_{e} m\end{array}\right)$. Then $x R_{e} y\left(R_{e} z R_{e}\right) m \subseteq N_{g}$. Since $R_{e} z R_{e}$ is an ideal of $R_{e}$, we have $K=\left(R_{e} z R_{e}\right) m$ is an $R_{e}$-submodule of $M_{g}$. Now, by Lemma 17, $x K \subseteq N_{g}$ or $y K \subseteq N_{g}$ or $x y M_{g} \subseteq N_{g}$. Hence, $x z R_{e} m \subseteq N_{g}$ or $y z R_{e} m \subseteq N_{g}$ or $x y M_{g} \subseteq N_{g}$. Thus, $x z \in\left(N_{g}:_{R_{e}} R_{e} m\right)$ or $y z \in\left(N_{g}:_{R_{e}} R_{e} m\right)$ or $x y R_{e} m \subseteq x y M_{g} \subseteq N_{g}$ that is $x y \in\left(N_{g}: R_{e} R_{e} m\right)$.

Lemma 19. Let $M$ be a $G$-graded $R$-module, $N$ be a graded $R$-submodule of $M$ and $I$ be an ideal of $R_{e}$. Assume that $g \in G$ such that $N_{g}$ is a 2-absorbing (strong 2-absorbing) $R_{e}$-submodule of $M_{g}$. If $x \in R_{e}$ and $m \in M_{g}$ such that $I R_{e} x R_{e} m \subseteq N_{g}(I x m \subseteq$ $\left.N_{g}\right)$, then $x m \in N_{g}$ or Im $\subseteq N_{g}$ or Ix $\subseteq\left(N_{g}: R_{e}\right.$ $\left.M_{g}\right)$.

Proof: Suppose that $x m \notin N_{g}$ and $I x \nsubseteq\left(N_{g}: R_{e}\right.$ $\left.M_{g}\right)$. Then there exists $y \in I$ such that $y x \notin\left(N_{g}:_{R_{e}}\right.$ $\left.M_{g}\right)$. Now, $y R_{e} x R_{e} m \subseteq N_{g}\left(y x m \in N_{g}\right)$ implies that $y m \in N_{g}$ as $N_{g}$ is a 2-absorbing (strong 2absorbing) $R_{e}$-submodule of $M_{g}$. We show that $I x \subseteq$ $N_{g}$. Let $z \in I$. Then $(y+z) R_{e} x R_{e} m \subseteq I R_{e} x R_{e} m \subseteq$ 
$N_{g}\left((y+z) x m \in I x m \subseteq N_{g}\right)$. Hence, $(y+z) m \in N_{g}$ or $(y+z) x \in\left(N_{g}:_{R_{e}} M_{g}\right)$. If $(y+z) m \in N_{g}$, then since $y m \in N_{g}$, we have that $z m \in N_{g}$. If $(y+z) x \in\left(N_{g}:_{R_{e}} M_{g}\right)$, then $z x \notin\left(N_{g}:_{R_{e}} M_{g}\right)$, on the other hand, $z R_{e} x R_{e} m \subseteq N_{g}\left(z x m \in N_{g}\right)$, so $z m \in N_{g}$. Therefore, $I m \subseteq N_{g}$.

Proposition 20. Let $M$ be a G-graded R-module and $N$ be a graded R-submodule of $M$. Assume that $g \in G$ such that $N_{g}$ is a 2-absorbing (strong 2-absorbing) $R_{e}$-submodule of $M_{g}$. Then $\left(N_{g}:_{M_{g}}\right.$ $I)=\left\{m \in M_{g}: I m \subseteq N_{g}\right\}$ is a 2-absorbing (strong 2-absorbing) $R_{e}$-submodule of $M_{g}$ for every ideal I of $R_{e}$.

Proof: Let $I$ be an ideal of $R_{e}$. Suppose that $x, y \in$ $R_{e}$ and $m \in M_{g}$ such that $x R_{e} y R_{e} m \subseteq\left(N_{g}: M_{g}\right.$ I) $\left(x y m \subseteq\left(\begin{array}{lll}N_{g} & :_{M_{g}} & I\end{array}\right)\right)$. So, Ix $R_{e} y R_{e} m \subseteq$ $N_{g}\left(\operatorname{Ixym} \subseteq N_{g}\right)$, and then $\left(I x R_{e}\right) R_{e} y R_{e} m \subseteq$ $I x R_{e} y R_{e} m \subseteq N_{g}$. Thus by Lemma 19, we have $\left(I x R_{e}\right) m \subseteq N_{g}$ or $\left(I x R_{e} y\right) \subseteq\left(N_{g}: R_{e} M_{g}\right)$ or ym $\in N_{g}$. If $y m \in N_{g}$, then Iym $\subseteq N_{g}$ and then $y m \in\left(N_{g}: M_{g} I\right)$ as required. If $I_{x} R_{e} y \subseteq$ $\left(N_{g}: R_{e} M_{g}\right)$, then $x y \in x R_{e} y \subseteq\left(\left(N_{g}: R_{e} M_{g}\right): R_{e}\right.$ $I)=\left(\left(N_{g}: M_{g} I\right):_{R_{e}} M_{g}\right)$. If $\left(I x R_{e}\right) m \subseteq N_{g}$, then $x m \in x R_{e} m \subseteq\left(N_{g}:_{M_{g}} I\right)$. Hence, ym $\in$ $\left(N_{g}:_{M_{g}} I\right)$ or $x m \in\left(N_{g}:_{M_{g}} I\right)$ or $x y \in\left(\left(N_{g}:_{M_{g}}\right.\right.$ $\left.I): R_{e} M_{g}\right)$ that ends the proof for 2-absorbing. For Ixym $\subseteq N_{g}$ and $N_{g}$ is strong 2-absorbing, it follows from Lemma 19 that $x y m \in N_{g}$ or Im $\subseteq N_{g}$ or Ixy $\subseteq\left(N_{g}: R_{e} M_{g}\right)$. If $x y m \in N_{g}$, then $x m \in N_{g}$ or $y m \in N_{g}$ or $x y \in\left(N_{g}:_{R_{e}} M_{g}\right)$. So, for $x m \in N_{g}$ it follows that $I x m \subseteq I N_{g} \subseteq N_{g}$ and we have $x m \in\left(N_{g}: M_{M_{g}} I\right)$. For ym $\in N_{g}$ it follows that Iym $\subseteq I N_{g} \subseteq N_{g}$ and we have $y m \in\left(N_{g}:_{M_{g}} I\right)$. For $x y \in\left(N_{g}:_{R_{e}} M_{g}\right)$, we have $x y \in\left(\left(N_{g}:_{R_{e}} M_{g}\right):_{R_{e}} I\right)=\left(\left(N_{g}:_{M_{g}} I\right):_{R_{e}} M_{g}\right)$. For $I m \subseteq N_{g}$, we have $m \in\left(N_{g}: M_{g} I\right)$ and hence $x m \in\left(N_{g}: M_{g} I\right)$. For $I x y \subseteq\left(N_{g}: R_{e} M_{g}\right)$, we have $x y \in\left(\left(N_{g}:_{R_{e}} M_{g}\right):_{R_{e}} I\right)=\left(\left(N_{g}:_{M_{g}} I\right):_{R_{e}} M_{g}\right)$ and $\left(N_{g}: M_{g} I\right)$ is a strong 2-absorbing $R_{e}$-submodule of $M_{g}$.

Proposition 21. Let $M$ be a graded R-module, $N$ and $K$ be graded $R$-submodules of $M$ such that $K \nsubseteq$ $N$. If $N$ is a graded 2-absorbing (graded strongly 2-absorbing) $R$-submodule of $M$, then $N \cap K$ is a graded 2-absorbing (graded strongly 2-absorbing) $R$ submodule of $K$.

Proof: By Lemma 13 and since $K \nsubseteq N, N \cap K$ is a proper graded $R$-submodule of $K$. Let $x, y \in h(R)$ and $k \in h(K)$ such that $x R y R k \subseteq N \cap K(x y k \in$ $N \cap K)$. Clearly, $x y K \subseteq K$ and $x k, y k \in K$. Furthermore, since $x R y R k \subseteq N \cap K \subseteq N(x y k \in$ $N \cap K \subseteq N$ ) and $N$ is a graded 2-absorbing (graded strongly 2-absorbing) $R$-submodule of $M, x y M \subset N$ or $x k \in N$ or $y k \in N$. So, $x y K \subseteq x y K \bigcap x y M \subseteq$ $N \cap K$ or $x k \in N \cap K$ or $y k \in N \cap K$. Hence, $N \cap K$ is a graded 2-absorbing (graded strongly 2absorbing) $R$-submodule of $K$.

Let $M$ be a $G$-graded $R$-module and $N$ be an $R$ submodule of $M$. Then $M / N$ may be made into a graded module by putting $(M / N)_{g}=\left(M_{g}+N\right) / N$ for all $g \in G$ (see [8]). Moreover, we have the following.

Lemma 22. ([1], Lemma 1.2) Let $M$ be a graded $R$-module and $K$ be a graded $R$-submodule of $M$. Suppose that $N$ is an $R$-submodules of $M$ such that $K \subseteq N$. Then $N$ is a graded $R$-submodule of $M$ if and only if $N / K$ is a graded $R$-submodule of $M / K$

Proof: Suppose that $N$ is a graded $R$-submodule of $M$. Clearly, $N / K$ is an $R$-submodule of $M / K$. Let $x+K \in N / K$. Then $x \in N$ and since $N$ is graded, $x=\sum_{g \in G} x_{g}$ where $x_{g} \in N$ for all $g \in G$ and then $(x+K)_{g}=x_{g}+K \in N / K$ for all $g \in G$. Hence, $N / K$ is a graded $R$-submodule of $M / K$. Conversely, let $x \in N$. Then $x=\sum_{g \in G} x_{g}$ where $x_{g} \in M_{g}$ for all $g \in G$ and then $\left(x_{g}+K\right) \in\left(M_{g}+K\right) / K=(M / K)_{g}$ for all $g \in G$ such that

$$
\begin{gathered}
\sum_{g \in G}(x+K)_{g}=\sum_{g \in G}\left(x_{g}+K\right)=\left(\sum_{g \in G} x_{g}\right)+K= \\
x+K \in N / K .
\end{gathered}
$$

Since $N / K$ is graded, $x_{g}+K \in N / K$ for all $g \in G$ which implies that $x_{g} \in N$ for all $g \in G$. Hence, $N$ is a graded $R$-submodule of $M$.

Proposition 23. Let $M$ be a graded R-module and $K$ be a graded $R$-submodule of $M$. Suppose that $N$ is an R-submodules of $M$ such that $K \subseteq N$. Then $N$ is a graded 2-absorbing (graded strongly 2absorbing) $R$-submodule of $M$ if and only if $N / K$ is a graded 2-absorbing (graded strongly 2-absorbing) $R$-submodule of $M / K$.

Proof: Suppose that $N$ is a graded 2-absorbing (graded strongly 2-absorbing) $R$-submodule of $M$. Then by Lemma $22, N / K$ is a proper graded $R$ submodule of $M / K$. Let $x, y \in h(R)$ and $m \in h(M)$ such that $x R y R(m+K) \subseteq N / K(x y(m+K) \subseteq$ $N / K)$. Assume that $a, b \in R$. Then $x a y b m+K=$ $x a y b(m+K) \subseteq x R y R(m+K) \subseteq N / K$, and then there exists $n \in N$ such that xaybm $+K=n+K$ which implies that $-n+x a y b m \in K \subseteq N$, and hence $x a y b m \in N$. So, $x R y R m \subseteq N$. (For 
the graded strong 2-absorbing case, $x y m+K=$ $x y(m+K) \subseteq N / K$ and then there exists $n \in N$ such that $x y m+K=n+K$, so $-n+x y m \in$ $K \subseteq N$, and then $x y m \in N$ ). Thus, $x m \in N$ or $y m \in N$ or $x y M \subseteq N$ as $N$ is a graded 2absorbing (graded strong 2-absorbing) $R$-submodule of $M$. Hence, $x(m+K) \in N / K$ or $y(m+K) \in$ $N / K$ or $x y(M / K) \subseteq N / K$. Therefore, $N / K$ is a graded 2-absorbing (graded strongly 2 -absorbing) $R$-submodule of $M / K$. Conversely, $N$ is a proper graded $R$-submodule of $M$. Let $x, y \in h(R)$ and $m \in$ $h(M)$ such that $x R y R m \subseteq N(x y m \in N)$. Then $m+K \in h(M / K)$ such that $x R y R(m+K) \subseteq N / K$ $(x y(m+K) \in N / K)$, and then $x(m+K) \in N / K$ or $y(m+K) \in N / K$ or $x y(M / K) \subseteq N / K$ as $N / K$ is a graded 2-absorbing (graded strongly 2 -absorbing) $R$-submodule of $M / K$. So, $x m \in N$ or $y m \in N$ or $x y M \subseteq N$, and hence $N$ is a graded 2-absorbing (graded strongly 2 -absorbing) $R$-submodule of $M$.

\section{Conclusion}

In this article, we study the concept of graded 2absorbing submodules over non-commutative rings. Also, we introduce the concept of graded strong 2absorbing submodules and show that in general if $R$ is not a commutative ring, then the concepts of graded 2 -absorbing and graded strong 2-absorbing submodules are not the same (Example 6). If $R$ is commutative, then the concept of graded 2-absorbing submodules coincides with that of the original definition introduced by Al-Zoubi and Abu-Dawwas in [3].

Acknowledgements: This research was partially supported by Yarmouk University. We are grateful for the insightful comments offered by the anonymous peer reviewers. The generosity and expertise of one and all have improved this article in innumerable ways and saved us from many errors.

\section{References:}

[1] R. Abu-Dawwas, Graded semiprime multiplication modules, accepted in Boletim da Sociedade Paranaense de Matemtica.

[2] R. Abu-Dawwas, M. Bataineh and M. AlMuanger, Graded prime submodules over noncommutative rings, Vietnam Journal of Mathematics, 46 (3) (2018), pp. 681-692.

[3] K. Al-Zoubi and R. Abu-Dawwas, On graded 2absorbing and weakly graded 2-absorbing submodules, Journal of Mathematical Sciences: Advances and Applications, 28 (2014), pp. 4560.
[4] K. Al-Zoubi, R. Abu-Dawwas and S. Ceken, On graded 2-absorbing and graded weakly 2absorbing ideals, Hacettepe Journal of Mathematics and Statistics, 48 (3) (2019), pp. 724-731.

[5] F. Farzalipour and P. Ghiasvand, On the union of graded prime submodules, Thai Journal of Mathematics, 9 (1) (2011), pp. 49-55.

[6] N. J. Groenewald and B. T. Nguyen, On 2absorbing modules over noncommutative rings, International Electronic Journal of Algebra, 25 (2019), 212-223.

[7] S. R. Naghani and H. F. Moghimi, On graded 2 -absorbing and graded weakly 2 -absorbing ideals of a commutative ring, Cankaya University Journal of Science and Engineering, 13 (2) (2016), pp. 11-17.

[8] C. Nastasescu and F. van Oystaeyen, Methods of Graded Rings, Lecture Notes in Mathematics, 1836, Springer-Verlag, Berlin, 2004. 